\title{
Towards Large-eddy Simulation of Turbulent Flows with Complex Geometric Boundaries Using Immersed Boundary Method
}

\author{
Xiaolei Yang ${ }^{1}$, Guo-Wei He. ${ }^{2}$ and Xing Zhang ${ }^{3}$ \\ LNM, Institute of Mechanics, Chinese Academy of Sciences, Beijing, 100190, China
}

\begin{abstract}
Approximate immersed boundary conditions for large-eddy simulation (LES) of turbulent flows in combination with immersed boundary method are proposed. The approximate immersed boundary conditions are based on the fluxes and the shear stresses in order to ensure the momentum conservation: the velocity boundary conditions are imposed to the convection terms and the shear stress boundary conditions imposed to the diffusion terms. On a body-fitted grid, they are exactly the same as the non-penetration and wall stress boundary conditions, for the immersed boundary method they are now the generalized off-wall boundary conditions. This strategy is tested in turbulent channel flows with the wall not being aligned with Cartesian grids. The results obtained show the promising of this strategy in simulations of high Reynolds number turbulent flows. The effects of different eddy-viscosity models used in conjunction with approximate immersed boundary conditions need to be further investigated.
\end{abstract}

\section{Nomenclature}

$\tilde{u}$

$=$ filtered velocity

$=$ time

$=$ global coordinate

$=$ density of the fluid

$=$ pressure

$=$ molecular viscosity

$=$ eddy viscosity

= body force

$=$ velocity in wall model

$=$ local coordinate

$=$ right-hand-side term in wall-layer model using boundary-layer equation

$=$ friction velocity defined as $\sqrt{\tau_{w} / \rho}$ where $\tau_{w}$ is the shear stress at the wall

$=$ half width of channel

$=$ Reynolds number defined as $\operatorname{Re}_{\tau}=u_{\tau} \delta / v$

= bulk velocity

$=$ the velocity at the centerline of the channel

$=$ Reynolds number defined as $\operatorname{Re}=(2 \delta) U_{b} / v$

$=$ Karman constant

\footnotetext{
${ }^{1}$ Graduate student, LNM, Institute of Mechanics, No.15 Beisihuanxi Road, Beijing 100190, China

2 Professor, LNM, Institute of Mechanics, No.15 Beisihuanxi Road, Beijing 100190, China

${ }^{3}$ Associate Professor, LNM, Institute of Mechanics, No.15 Beisihuanxi Road, Beijing 100190, China
}

American Institute of Aeronautics and Astronautics 


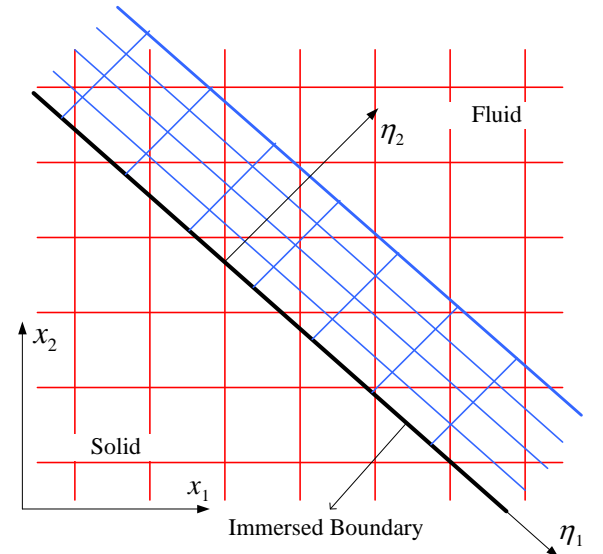

Figure 1. Two sets of grids for IB method with wall-layer model. The red one is for LES, the blue one is for boundary-layer equations. $\begin{array}{ll}y_{w}^{+} & =\text {distance to the wall normalized defined as } y_{w}^{+}=y_{w} u_{\tau} / v \\ \mathrm{~A} & =\text { constant in damping function }\end{array}$

\section{Introduction}

Immersed boundary (IB) method [1] exhibits its advantage in simulating flows with complex geometric boundaries and moving boundaries [2, 3] and it has been successfully applied to laminar flows. In industrial and environmental applications, the Reynolds numbers of flows are usually very high. Large-eddy simulation (LES) can be used to capture the large eddies and the unsteadiness of these flows at high Reynolds numbers. LES has been successfully applied to free shear flows at moderate Reynolds numbers. But it is less successful for wallbounded flows at high Reynolds number due to the grid resolution requirement in the near-wall region. In order to resolve the wall-layer structures, the number of grid needs to be proportional to $\operatorname{Re}_{\tau}{ }^{2}$ [4] in the near-wall region. Instead of resolving the wall-layer, one alternative is to use the wall-layer model. The approximate boundary conditions for LES are obtained from the wall models, which represents the effects of the wall-layers on the outer flows. Generally, the shear stress boundary conditions are used for the streamwise and spanwise velocity components, and the non-penetration boundary conditions are used for the wall-normal velocity component. There are different ways to calculate the wall shear stress on the wall. Deardorff [5] and Schumann [6] used the equilibrium laws to calculate the shear stress. Balaras and Benocci [7] proposed to use boundary-layer equations to calculate the shear stress. Wang and Moin develop the boundary layer equations to calculate trailing edge noise [8]. The relevant reviews can be found in the papers by Cabot and Moin [9], Piomelli and Balaras [10] and Piomelli [11]. Most of the previous studies focus on wall-layer model for body-fitted grid. There is little work on its combination with IB method. Choi et al. [12] used the power law to reconstruct the tangential velocity near the wall. Log-law was used by Chester et al. [13] to calculate the shear stress. In their work, turbulent flows past fractal trees were simulated. Tessicini et al. [14] used a simplified version of the boundary-layer equations named as the equilibrium stress balance model for LES. Roman et al. [15] proposed to model the wall shear stress by imposing a RANS-like viscosity at the immersed boundary.

In this paper, we propose a new strategy in the IB method for LES with wall models. A LES is used to simulate the outer flows using Cartesian grids and a wall model based on boundary layer equation (BLE) is used for inner flows. The sub-domain for LES and BLE overlap each other. In wall models, the wall stress and non-penetration boundary conditions are usually used to ensure the shear stress and impermeability at the wall. For the LES on the Cartesian grids in this work, the shear stress and flux boundary conditions imposed. However, it is different from the conventional velocity boundary conditions [16]. This paper is organized as follows: In section 2, a new strategy for immersed boundary method is introduced. In section 3, this new strategy is tested against the fully developed turbulent channel flows. A summary is drawn in section 4.

\section{Immersed boundary method for LES with wall-layer models}

In the LES of wall-bounded turbulent flows in combination with immersed boundary method, outer flows are simulated using LES on Cartesian grids with immersed boundary methods while inner flows in near- wall regions are simulated using wall models on either body-fitting or non-body-fitting grids. Coupling the LES with the wall models is made in the overlapping region. In the simplest case, the boundary conditions for the LES are reconstructed by interpolation of the velocity fields from the wall models, while the LES offers the boundary conditions for wall models. The inner domains are usually extended to the log-law regions so that the interpolation can be better carried out on the LES regions.

In the LES, the filtered Navier-Stokes equations with a body force are given by

$$
\frac{\partial \tilde{u}_{i}}{\partial t}+\frac{\partial \tilde{u}_{i} \tilde{u}_{j}}{\partial x_{j}}=-\frac{1}{\rho} \frac{\partial p}{\partial x_{i}}+\frac{\partial}{\partial x_{j}}\left[\left(v+v_{t}\right)\left(\frac{\partial \tilde{u}_{i}}{\partial x_{j}}+\frac{\partial \tilde{u}_{j}}{\partial x_{i}}\right)\right]+f_{i}
$$




$$
\frac{\partial \tilde{u}_{i}}{\partial x_{i}}=0
$$

They are discretized on the Cartesian grids as shown in figure 1 . The $x_{1}, x_{2}, x_{3}$ represent the streamwise, crosswise and spanwise coordinates in a global coordinate system, respectively. The grid points in the Cartesian coordinate system are classified into three types: the fluid points, the solid points and the forcing points as shown in figure 2. The forcing points represent the boundary for LES by which outer flows are simulated. For wall-resolved simulations, the velocities at the forcing points are obtained by interpolation of the velocities at the surrounding fluid points and the ones at the solid boundaries.

In near-wall region, the turbulent boundary layer equations are used in the following form

$$
\begin{gathered}
\frac{\partial}{\partial \eta_{2}}\left(v+v_{t}\right) \frac{\partial \bar{u}_{i}}{\partial \eta_{2}}=F_{i}, \quad(i=1,3) \\
\frac{\partial \bar{u}_{i}}{\partial \eta_{i}}=0
\end{gathered}
$$

where

$$
F_{i}=\frac{\partial \bar{u}_{i}}{\partial t}+\frac{\partial \bar{u}_{i} \bar{u}_{j}}{\partial \eta_{j}}+\frac{1}{\rho} \frac{\partial p}{\partial \eta_{i}} .
$$

The $\eta_{1}, \eta_{2}, \eta_{3}$ represent the wall-tangent, wall-normal and spanwise coordinates in a local coordinate system, respectively. The velocities in the wall-tangent $(\mathrm{i}=1)$ and the spanwise $(\mathrm{i}=3)$ directions are solved from equation (3). The wall-normal velocity ( $\mathrm{i}=2$ ) is determined by the continuity equation (4). The eddy viscosity in equation (3) is determined by the mixing-length eddy viscosity model with near-wall damping

$$
\frac{v_{t}}{v}=\kappa y_{w}^{+}\left(1-e^{-y_{w}^{+} / A}\right)^{2}
$$

where $y_{w}^{+}=y_{w} u_{\tau} / v$ is the distance to the wall in wall units, $\kappa$ is the model coefficient, and $A=19$. Since $F_{i=1,3}$ are treated as source term in the wall model, equations (3)and (4) are only integrated in the wall-normal direction with its refined mesh as shown in figure 1 . The pressure gradients are provided by the outer flows, so that no Poisson equation is required to determine the pressure. In this paper, only the equilibrium stress balance model

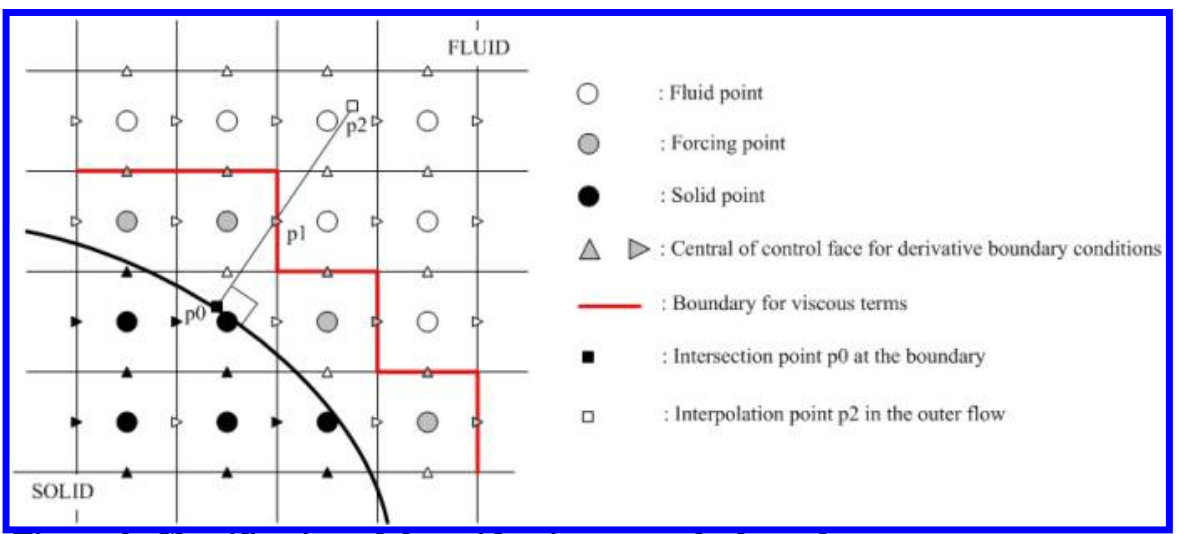

Figure 2. Classification of the grid points near the boundary with $F_{i}=0$ is considered.

The velocities and their gradients at the forcing points can be obtained from either LES or wall models: the velocities at the forcing points are interpolated from the velocities at the surrounding points; the shear stresses at the forcing points are interpolated from the wall stresses at the wall and the shear stresses at the interface of LES and wall model, and then, the

3

American Institute of Aeronautics and Astronautics 
velocity gradients at the forcing points can be obtained from the shear stresses at the same forcing points. In the LES with near wall resolution, the interpolation is a reasonable approximation. However, in the LES without wall resolution, the grid points for interpolation have to be located on the walls and the LES boundaries.

In the present work, the forcing points serve as the boundaries for LES. Since the velocities and their derivatives at the forcing points can be obtained, it is problematic how to choose appropriate boundary conditions for the LES. We propose to use the velocity boundary conditions for the convection term and the velocity gradient boundary conditions for the dissipation term respectively. Conventionally, only one type of boundary conditions, either velocities or velocity gradients, is used for both convection and dissipation terms. However, in the present IB method, we use different boundary conditions for the convection and dissipation terms. The velocity boundary conditions for the convection term ensure the correct representation of fluxes and the velocity gradient boundary conditions ensure the correct representation for shear stresses. If the forcing points are coincident with the solid points, the proposed boundary conditions are the same as the non-penetration and wall stress conditions. The latter has been successfully used to the wall modeling on body-fitting grids.

\section{Numerical results}

The performance of the present implementation of wall model in IB method is examined by fully developed turbulent channel flows at $\operatorname{Re}_{r}=1000,2000$ and 4000 on a coarse grid. The channel size is $2 \pi \delta \times 2 \delta \times \pi \delta$ in the streamwise ( $\mathrm{i}=1)$, wall-normal $(\mathrm{i}=2)$ and spanwise directions $(\mathrm{i}=3)$, where $\delta$ is the channel half-width. The number of the computational cell in the channel is 32 for the streamwise and spanwise directions. The grid conforms to the top wall while the bottom wall is shifted so that no grid line coincides with it. In the wall-normal direction, three types of grid are used. For grid 1 and grid 2, the number of the computational cell in the wall-normal direction is 58 with approximate 48 cells located in the channel flow region, which are used for simulations with $\operatorname{Re}_{\tau}=2000$ and 4000. In grid 1 , the distance of the first off-wall gird point from the lower wall is $8 \times 10^{-4}$ for the velocity components parallel to the wall. In grid 2, the distance from the wall to the first off-wall point is $1.2 \times 10^{-2}$. In grid 3 , the total cell number in the wall-normal direction is 45 with approximate 35 cells in the channel flow region. The distance from the wall to the first off-wall point is $3.92 \times 10^{-2}$ for gird 3 . The boundary conditions used in wall model are applied directly at the top wall. At the bottom wall, the procedure proposed in this work is used to apply the boundary conditions. The dynamic Smagorinsky model is used in the large-eddy simulation. The model constant $\kappa$ in mixing-length eddy viscosity model is chosen as 0.41 .

Table 1 Errors of wall shear stress and centerline velocity for channel flow

\begin{tabular}{|l|c|c|c|c|}
\hline & $\operatorname{Re}_{\tau}$ & Error & $U_{0} / U_{b}$ & error \\
\hline $\mathrm{Re}=1000$ on grid 3 & $975.23(1015.63)$ & $3.98 \%$ & $1.12(1.16)$ & $3.45 \%$ \\
\hline $\mathrm{Re}=2000$ on grid 2 & $1960.20(2032.14)$ & $3.54 \%$ & $1.10(1.13)$ & $2.65 \%$ \\
\hline $\mathrm{Re}=4000$ on grid 1 & $4303.75(4196.60)$ & $2.55 \%$ & $1.07(1.10)$ & $2.73 \%$ \\
\hline $\mathrm{Re}=4000$ on grid 2 & $4023.47(4065.34)$ & $1.03 \%$ & $1.08(1.10)$ & $1.82 \%$ \\
\hline $\mathrm{Re}=4000$ (VEL_BC) on grid 1 & $5059.11(4064.17)$ & $24.48 \%$ & $1.09(1.10)$ & $0.91 \%$ \\
\hline $\mathrm{Re}=4000$ (VEL_BC) on grid 2 & $3850.52(4064.07)$ & $5.25 \%$ & $1.08(1.10)$ & $0.91 \%$ \\
\hline
\end{tabular}

The numerical results are summarized as follows. Firstly, the results from simulations at $\operatorname{Re}_{\tau}=1000,2000$ and 4000 on the gird of $y^{+}(1)=48$ are presented. Secondly, the effect of the distance of the first off-wall grid point to the wall is examined by simulating channel flows at $\operatorname{Re}_{\tau}=4000$ on the grid of $y^{+}(1)=3.2$. Lastly, the present procedure is compared with another method of using wall-modeling in conjunction with the IB method to simulate the channel flow at $\operatorname{Re}_{\tau}=4000$ on the grid 1 and grid 2. In that method, boundary condition for the wall-parallel velocities (other than the stresses) from the solution of wall-model is used. The wall-normal velocity is also interpolated from the outer flow the value at the wall as in the present procedure. This procedure is named as "VEL_BC".

Table 1 gives the errors in Reynolds number based on the computed friction velocity and centerline velocity relative to the approximate formulae as follows

$$
\operatorname{Re}_{\tau} \approx 0.09 \operatorname{Re}^{0.88}
$$




$$
U_{0} / u_{\tau} \approx 5 \log _{10} \operatorname{Re}
$$

where Re is based on the bulk velocity and the whole channel width, $U_{0}$ is the centerline velocity. By using the bulk velocity from the simulation, the Reynolds number based on friction velocity and the ratio of centerline velocity to the bulk velocity can be computed from formulae (7) and (8). It can be seen that all the errors are within 5\% except
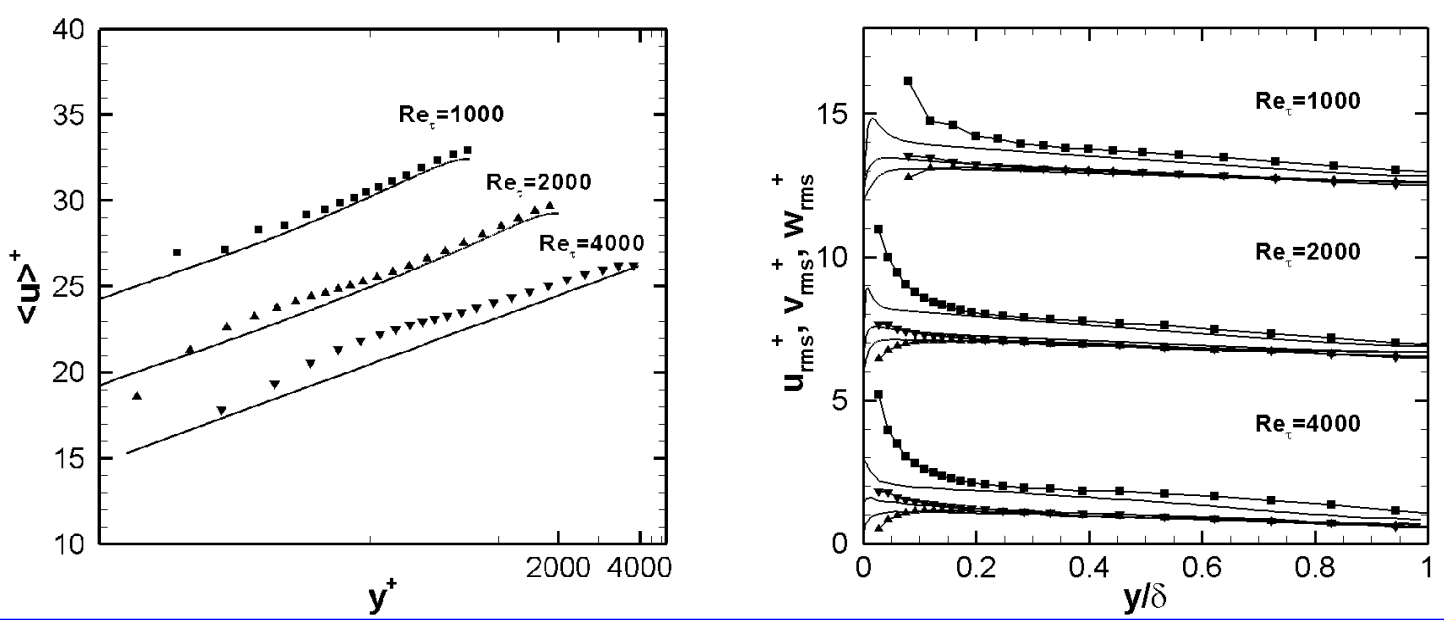

Figure 3. Turbulence statistics for channel flows with Reynolds number 1000, 2000 and 4000 at grid 2 with $y^{+}(1)=48$. Left plot: mean velocity profiles; the mean velocity profiles are skipped by five units for clarity in visualization. Right plot: root-mean-square (RMS) velocity fluctuations. The References are in solid line. For Reynolds number at 1000 and 2000, the reference data is from the DNS results of Hoyas and Jiménez [17]. The reference data for Reynolds number at 4000 is from the LES data of [18].

for the wall shear stress predicted by using the boundary conditions of 'VEL_BC'.

Fig. 3 gives the results from turbulent channel flows at $\mathrm{Re}_{\tau}=1000,2000$ and 4000 . The values at the first offwall point are not shown in the plots for clarity since the velocities at the forcing points are simply interpolated from the velocities at the second-off-wall grid and the velocities at the wall. Left figure shows the mean velocity profiles. It can be seen that the mean velocity profiles show good agreement with the DNS data or the logarithmic law. Right plot shows the RMS velocity fluctuations. It is seen that the present wall-layer model poorly predicts the RMS velocity fluctuations near the wall. In the outer flow, the RMS velocity fluctuations in wall-normal and spanwise directions show good agreement with the reference data $[17,18]$. For the streamwise component, the predicted results are a little higher than the reference data [17,18]. 

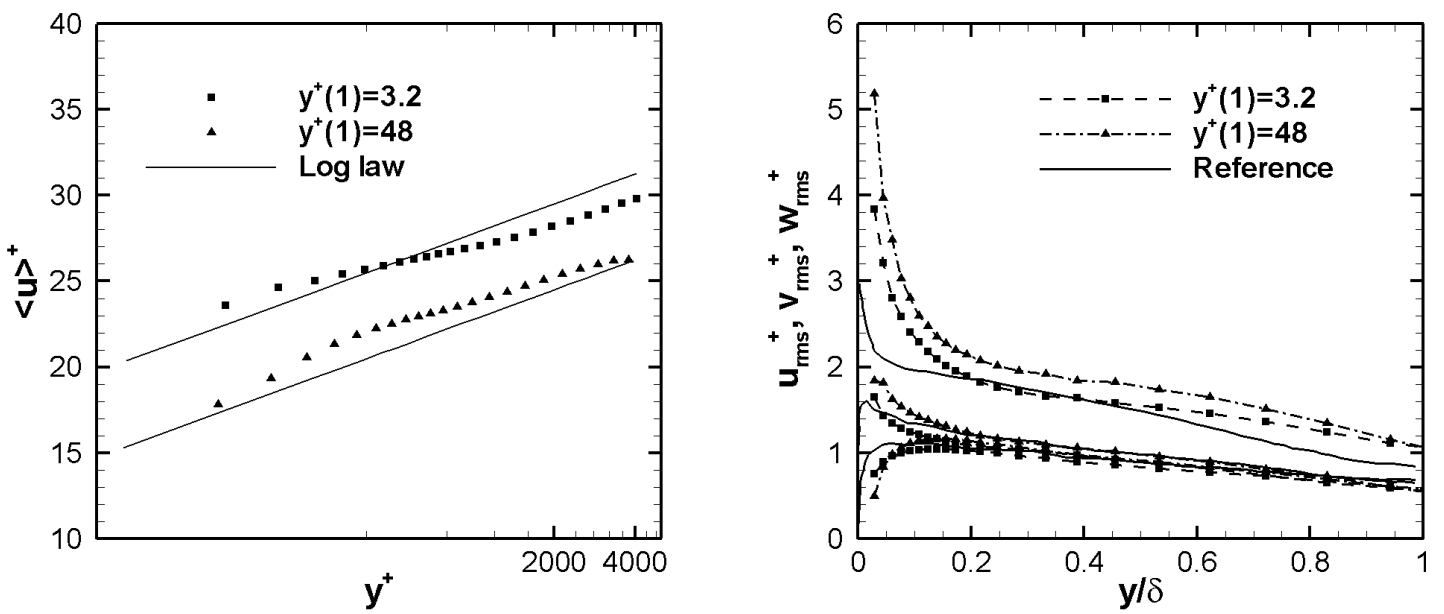

Figure 4. Comparison of the turbulence statistics for channel flow at $\mathrm{Re}_{\tau}=4000$ at different grids. Left: Mean velocity profiles compared with the log law. The mean velocity profiles are skipped by five units for clarity in visualization. Right: RMS velocity fluctuations compared with LES data of [18] shown with solid lines.

Fig. 4 compares the results of turbulent channel flows at $\mathrm{Re}_{\tau}=4000$ with different grid near the lower wall. From the left plot, it can be seen that the mean velocity profiles agree well with the log-low, independent of the distance of the first off-wall grid from the wall. The right plot shows the RMS velocity fluctuations. For the RMS velocity fluctuations, results for the wall-normal and spanwise components on the two grids with $y^{+}(1)=3.2$ and $y^{+}(1)=48$ are very close to each other. For the streamwise components, the two results look very similar with small discrepancies.

Fig. 5 shows the turbulence statistics from the simulations using the "VEL_BC" method. The left plot shows the mean velocity profile. For the results from the grid 2 with $y^{+}(1)=3.2$, both the mean velocity profile and the RMS velocity fluctuations show large discrepancies from the reference data [18] since the friction velocity is too highly over-predicted. For the results from the grid 1 with $y^{+}(1)=48$, the mean velocity profiles show good agreement with the log law. The wall-normal and spanwise RMS velocity fluctuations components show good agreement with the reference in the outer flows. The streamwise component is over-predicted, especially near the wall. In general, the results in Fig. 5 show that the performance of the "VEL_BC" method depends on the distance of the first offwall grid sensitively. The results are acceptable when the first off-wall points are in the logarithmic layer. The results are very poor when the first off-wall points are beneath the logarithmic layer.

\section{Summary}

Approximate immersed boundary conditions in the framework of immersed boundary method are proposed for Large Eddy Simulation (LES) of high Reynolds number turbulent flows. In an 'inner layer' adjacent to the boundary, the Boundary Layer Equation (BLE) is solved on a body-fitting grid. The solution of the BLE serves as the wall model for the outer layer, where LES is performed in conjunction with the immersed boundary method.

In the immersed boundary method, a linear reconstruction near the wall is sufficient to impose the correct boundary condition when the grid resolution is high enough to resolve the wall-layer. However, this is not true for the case in which the grid is substantially coarse such that the first off-wall point is typically located in logarithmic region. In this work, we proposed to enforce the non-penetration condition of velocity in LES by using the conventional method of linear extrapolations. This provides the boundary condition only for the convection term, while for the diffusion term, a boundary condition of shear stress is provided by the BLE. 

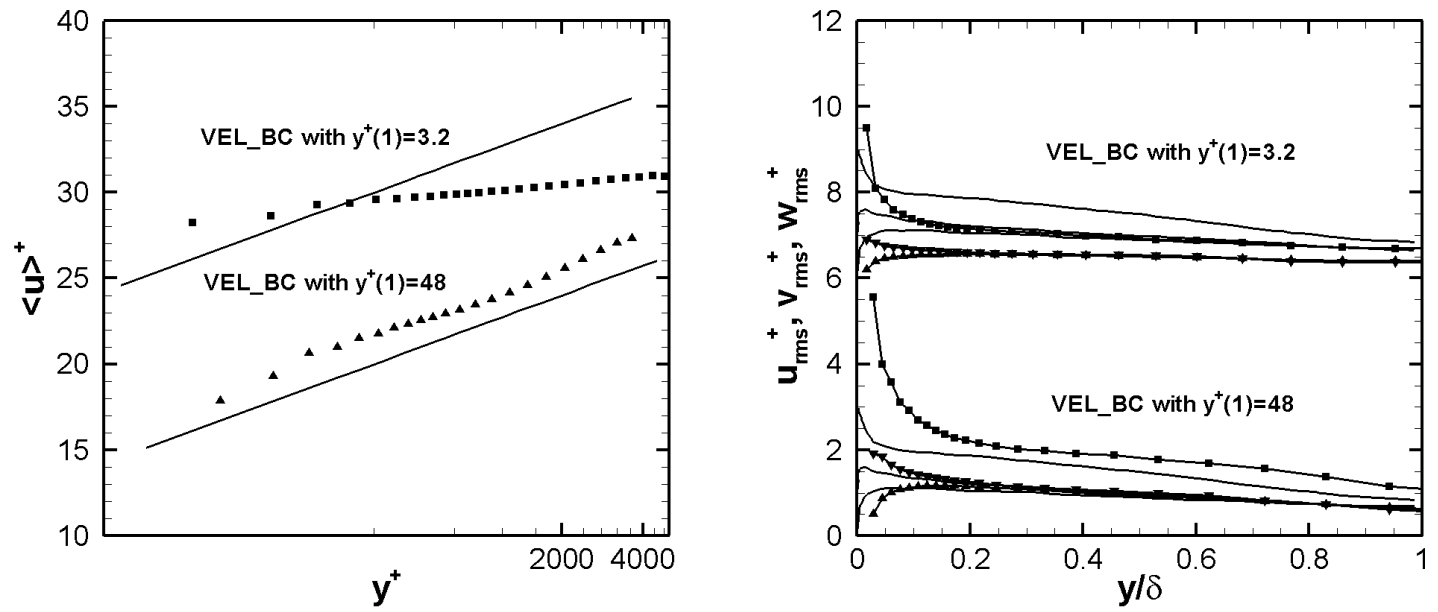

Figure 5. Turbulence statistics for channel flows by using velocity boundary conditions for LES. Left: Mean velocity profiles with the log law. The mean velocity profiles are skipped by ten units for clarity in visualization. Right: RMS velocity fluctuations compared with LES data of [18] shown with solid lines. The RMS velocity fluctuations are skipped by six units for clarity in visualization.

The ability of the present method is primarily tested by simulating the fully developed turbulent channel flows. The results obtained show its promise in simulating turbulent flows of high Reynolds numbers. Our test on the flows over periodic hills finds the discrepancies in the predictions of flows with separation. Those are probably due to the oversimplifications in the BLE used in the present work. More sophisticated wall-layer models, presumably, could be the remedy. Other issues which are also crucial to the present approach but are not discussed are the overlapping width between the two layers and the eddy viscosity model used. More works are required to investigate their influence on the prediction.

\section{Acknowledgments}

This work was supported by Chinese Academy of Sciences under the Innovative Project "Multi-scale modeling and simulation in complex systems" (KJCX-SW-L08), "Mathematical modeling of complex system" (KJCX3SYW-S01), National Basic Research Program of China (973 Program) under Project No. 2007CB814800, and National Natural Science Foundation of China under Project Nos. 10325211, 10628206, 10732090 and 10872201.

\section{References}

[1] R. Mittal and G. Iaccarino, "Immersed boundary method, ” Ann. Rev. Fluid Mech. 37 (2005) pp 239-261. .

[2] N. Zhang and Z.C. Zheng, An improved direct-forcing immersed-boundary method for finite difference applications Journal of computational Physics Vol. 221 (2007) pp.250-268

[3] X. Yang, X. Zhang, Z. Li, G.-W. He, “A smoothing technique for discrete delta functions with application to immersed boundary method in moving boundary simulations”, J. Comput. Phys., Vol. 228, (2009), pp 7821-7836.

[4] J. S. Baggett, "Some modeling requirements for wall models in large eddy simulation”, CTR Annu. Res. Briefs, (1997), pp 123-134.

[5] JW. Deardorff, “A numerical study of three-dimensional turbulent channel flow at large Reynolds number”, J. Fluid Mech., Vol. 41, (1970), pp 453-480.

-[6] U. Schumann, "Subgrid-scale model for finite difference simulation of turbulent flows in plane channels and annuli”, J. Comput. Phys., Vol. 18, (1975), pp 376-404

[7] E. Balaras, C. Benocci, "Subgrid-scale models in finite-difference simulations of complex wall bounded flows", AGARD CP, Neuilly-Sur-Seine, France: AGARD, 551, (1994), pp 2.1-2.5.

[8] M. Wang and P. Moin, "Dynamic wall modeling for large-eddy simulation of complex turbulent flows”, Phys. Fluids, Vol. 14, No. 7, (2002), pp 2043-2051

[9] W. Cabot and P. Moin, “Approximated wall boundary conditions in the large-eddy simulation of high Reynolds number flow”, Flow, Tubul. Combust. Vol. 63, (1999), pp 269-291. 
[10] U. Piomelli and E. Balaras, “Wall-layer models for large-eddy simulations”, Annu. Rev. Fluid Mech., Vol. 34, (2002), pp 349-374.

[11] U. Piomelli, “Wall-layer models for large-eddy simulations”, Progress in Aerospace Sciences, Vol. 44, (2008), pp 437-446.

-[12] J. Choi, R. C. Oberoi, J. R. Edwards and J. A. Rosati, “An immersed boundary method for complex incompressible flows”, J. Comput. Phys., Vol. 224, (2007), pp 757-784.

[13] S. Chester, C. Meneveau and M. B. Parlange, "Modeling turbulent flow over fractal trees with renormalized numerical simulation”, J. Comput. Phys., Vol. 225, (2007), pp 427-448.

[14] F. Tessicini, G. Iaccarino, M. Fatica, M. Wang and R. Verzicco, "Wall modeling for large-eddy simulation using an immersed boundary method”, CTR Annu. Res. Briefs, (2002), pp 181-187.

-[15]F. Roman, V. Armenio and J. Frohlich, “A simple wall-layer model for large eddy simulation with immersed boundary method”, Phys. Fluids, Vol. 21, (2009), 101701.

[16] J.M. Yang and E. Balaras, An immersed-boundary formulation for large-eddy simulation of turbulent flows interacting with moving boundaries, Journal of Computational Physics, Vol. 215 (2006) pp 12-40.

[17] S. Hoyas and J. Jiménez, "Scaling of the velocity fluctuations in turbulent channels up to Re_=2003”, Phys. Fluids, Vol. 18, (2006), 011702.

[18] A. G. Kravchenko, P. Moin and R. Moser, "Zonal Embedded Grids for Numerical Simulations of Wall-Bounded Turbulent Flows”, J. Comput. Phys., Vol 127, (1996), pp 412-423. 